\title{
A Survey on Reactive Routing Protocols of the Mobile Ad hoc Networks
}

\author{
Gurleen Kaur Walia \\ AP at Guru Nanak Dev \\ University, Regional Campus, \\ Jalandhar
}

\begin{abstract}
Mobile Ad hoc Networks (MANET) are wireless networks without an infrastructure, which are usually set up on a temporary basis to serve a particular purpose within a specific period of time. An efficient routing protocol for MANET has become a necessary and important issue to be considered before deploying any mobile network. This paper presents a comparison among the reactive routing protocols: Ad-hoc OnDemand Distance Vector Routing (AODV), Dynamic Source Routing (DSR) and Temporally Ordered Routing Algorithm (TORA). The effects on the routing efficiencies with a special focus on the mobility and node density using end to end delay, throughput, retransmission attempts and data dropped as indices of performance evaluation for FTP traffic were observed by using OPNET 14.5 modeler as simulation tool. Based on the observations from literature and empirical study conducted using OPNET, it is found that among the three protocols, no single protocol can successfully provide optimum efficiency in different MANET scenarios.
\end{abstract}

\section{General Terms}

Performance analysis, simulation, graphs.

\section{Keywords}

Ad hoc Network, Routing Protocols, Throughput, End to end delay, data dropped.

\section{INTRODUCTION}

Mobile Ad-hoc Network (MANET) is a wireless system comprising of mobile nodes that dynamically establishes the network in the absence of fixed infrastructure. It is usually referred to a decentralized autonomous system [1], [8]. MANET nodes are equipped with wireless transmitters and receivers. The term "ad hoc" tends to imply "can take different forms" and can be "stand-alone, mobile or networked". Each node acts as a router to find out the optimal path to forward a packet.

As nodes are mobile, entering and leaving the network, the topology of the network changes continuously. Due to the infrastructure less, self-configuring network property, MANET has wide application in industrial and commercial field involving cooperative mobile data exchange, inexpensive alternatives or enhancement to cellular-based mobile network infrastructures. MANET has potential applications in the locations where setting of infrastructured networks is not possible.
MANET gradually exploited the wireless communication world as the common means for human communication. This challenged the researchers around the world to enforce their research in developing MANET. In such advanced communication network, routing plays a key role, as it is one of the major aspects to route the data in network. Many researchers have proposed different protocols so far. This exploration of wireless devices lead the path to focus our study on the large networks where hosts involved in the network engage to communicate each other in Ad hoc fashion.

\section{RELATED WORK}

Broch et al. [2], conducted experiments with DSDV, TORA, DSR and AODV. They used a constant network size of 50 nodes, 10 to 30 traffic sources, seven different pause times and various movement patterns. Packet delivery fraction (PDF), number of routing packets and distribution of path lengths were used as performance metrics. They extended $n s$ 2 discrete event simulator [3], developed by the University of California at Berkeley and the VINT project [4], to correctly model the MAC and physical-layer behavior of the IEEE 802.11 wireless LAN standard.

Juan-Carlos Cano and Pietro Manzoni [5] concentrated on the energy consumption issues of routing protocols. They presented a performance comparison of the DSR, AODV, TORA and DSDV routing protocols with respect to energy consumption.

Ehsan and Uzmi [6] presented the performance comparison of DSDV, AODV, DSR and TORA based on simulations performed using network simulator-2. Three metrics: normalized routing overhead, packet delivery fraction and average end to end delay, were used to measure performance.

Karthikeyan et al. [7] studied the Reactive protocols, DSR and AODV as well as a Proactive Protocol, DSDV and their characteristics with respect to different mobility were analyzed based on packet delivery fraction, routing load, endto-end delay, number of packets dropped, throughput and jitter using Network Simulator (ns-2).

\section{MANET ROUTING PROTOCOLS}

\subsection{Classification of Routing Protocols}

The Ad-hoc routing protocols can be divided into three categories: Table-Driven Routing Protocols, On-Demand Routing Protocols and Hybrid Routing Protocols. 


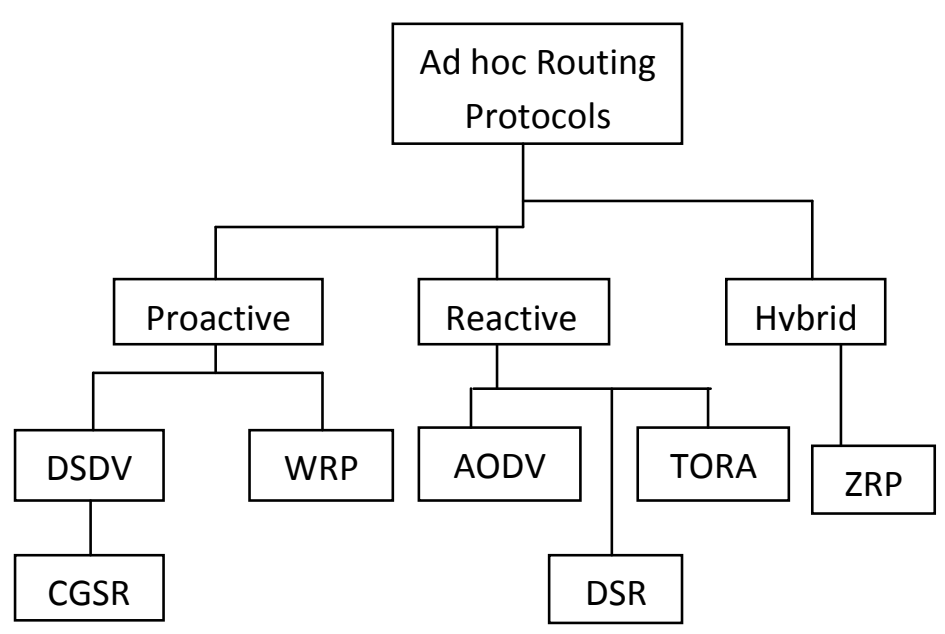

Figure 1. Classification of Routing Protocols

\subsection{Reactive Routing Protocols}

\subsubsection{Dynamic Source Routing (DSR) [9]:}

The main feature of the DSR protocol is source routing. It is specially designed for multi-hop ad-hoc networks and reduces the utilized bandwidth by eliminating messages, which are periodic. In DSR, a complete list of the nodes is included in the data packet, to which they should be forwarded to. This protocol has two major basic mechanisms including "route discovery", and "route Maintenance". During route discovery, a source node broadcasts a RREQ message, and each intermediate node receiving this packet will rebroadcast it, unless it is either the destination or has the route to destination information in its route cache. Such a node will send a RREP message to the source. The route, which carries the RREP packet, is cached in the source node for future use. If a link failure occurs, then a route error packet (RERR) will be sent to the source to notify it. The source node then removes that routes consisting failed link from its cache and if there is a new route to that destination in its cache, it will replace it with the previous one. Otherwise, it will reinitiate route discovery.

\subsubsection{Ad-hoc On-Demand Distance Vector} Routing (AODV) [10], [15]:

AODV is an on demand algorithm, meaning that it builds routes between nodes only as desired by source nodes like DSR, via similar Route Discovery process. This routing algorithm broadcasts by creating routes on an on-demand basis, as opposed to maintaining a complete list of routes as in proactive algorithm. A path discovery is initiated when a route to a destination does not exist. Broadcast is used for route request. Link failure notification is sent to the upstream neighbors and this algorithm requires symmetric links.

Ad hoc on demand distance vector, as the name suggests is a routing protocol designed to build routes between nodes only when demanded by source nodes. Routes are maintained as long as they are needed by the sources. Sequence numbers are used in order to maintain the routes up to date. Routes are built using route request/reply messages. When a source node wishes to communicate with a destination for which it does not have a route, it broadcasts a route request (RREQ) packet across the network. A node receiving the packet updates the information about the source node and sets backward pointers to the source node in the route tables. A node will then unicast a route reply (RREP) if it is the destination or if it has a route to the destination with a greater or equal sequence number to that of the RREQ message. Otherwise the node rebroadcasts the RREQ packet. If it has already been processed it is then being discarded. When a RREP propagates back to the source, nodes set up forward pointers to the destination. Once the source node receives the RREP, which includes the sequence number of the destination and the hop count to reach it, data packets can be sent to the destination using the forward route entries of the intermediate nodes. If a RREP is received later with a greater or equal sequence number and shorter hop count, the better route can be used. Timeouts are used in order to keep the routes active for certain periods of time. In the case a link breaks while a route is active, the node upstream of the breakage will propagate a route error (RERR) message to the source node to inform that the destination is unreachable. The source node can then reinitiate RREQ if desired. Additionally sequence numbers are used in order to maintain the freshness of routing information and loop avoidance.

\subsubsection{Temporally Ordered Routing Algorithm (TORA) [12], [13]:}

TORA is a distributed routing protocol which is designed to discover routes on demand. It provides multiple routes to a destination, establish routes quickly and minimize communication overhead. Route optimality (shortest-path routing) is considered of secondary importance and longer routes are often used to avoid the overhead of discovering newer routes. TORA builds and maintains a Directed Acyclic Graph (DAG) rooted at a destination. No three nodes may have the same height. Information may flow from nodes with higher heights to nodes with lower heights. Information can therefore be thought of as a fluid that may only flow downhill. By maintaining a set of temporally ordered heights at all times, TORA achieves loop-free multipath routing, as information cannot 'flow uphill' and so cross back on itself. The key design concept of TORA is localization of control messages to a very small set of nodes near the occurrence of a topological change. To accomplish this, nodes need to maintain the routing information about adjacent (one hop) nodes. The protocol performs three basic functions:

- Route creation

- Route maintenance

- Route erasure

During the route creation and maintenance phases, nodes use a height metric to establish a directed acyclic graph (DAG) rooted at destination. Thereafter links are assigned based on the relative height metric of neighboring nodes. During the times of mobility the DAG is broken and the route maintenance unit comes into picture to reestablish a DAG routed at the destination. Timing is an important factor for TORA because the height metric is dependent on the logical time of the link failure. TORA's route erasure phase is essentially involving flooding a broadcast clear packet (CLR) throughout the network to erase invalid routes.

\section{Simulation Design}

OPNET (Optimized Network Engineering Tool) Modeler 14.5 is used for the design and implementation of this work. OPNET provides virtual network communication environment and is prominent for the research studies, network modeling and engineering, $\mathrm{R} \& \mathrm{D}$ Operation and performance analysis. 
5. Performance Metrics: The following metrics have been considered to make the comparative study of these routing protocols through simulation.

5.1 End-to-end delay: This metric represents average end-to-end delay and indicates how long it took for a packet to travel from the source to the application layer of the destination. It is measured in seconds.

\subsection{Throughput: Throughput refers to how much data can} be transferred from one location to another in a given amount of time. Unit of throughput is bits/sec or packets/sec. Throughput in aspect of MANET is affected due to topology change, bandwidth etc.

5.3 Retransmission Attempts: This metric represents the number of retransmissions due to some failures in the network. It is measured in terms of packets.

\subsection{Data Dropped}

The parameters that have been used in the following experiments are summarized in Table 1.

Table 1. Simulation Parameters

\begin{tabular}{|c|c|}
\hline Attribute & Value \\
\hline Simulator & OPNET 14.5 \\
\hline Protocols Studied & AODV, DSR, TORA \\
\hline Scenario Size & $1000 \mathrm{~m} \times 1000 \mathrm{~m}$ \\
\hline Nodes & 10,100 \\
\hline Node Mobility (m/s) & FTP \\
\hline Traffic Type & Random Waypoint Model \\
\hline Node Movement Model & 0.005 \\
\hline Transmit Power (W) & 3 minutes \\
\hline Simulation Time & \\
\hline
\end{tabular}

\section{SIMULATION RESULTS \& OBSERVATIONS}

The simulation results are shown in the form of graphs, which show comparison between the three protocols by varying different numbers of sources on the basis of above mentioned metrics.

The simulation results are shown in the form of graphs, which show comparison between the three protocols by varying different numbers of sources on the basis of above mentioned metrics.

\subsection{End-to-end Delay:}

\subsubsection{Number of Nodes: 10, 100 and Mobility: $10 \mathrm{~m} / \mathrm{s}$}

In Figure 2, end-to-end delay parameter is simulated here for 10 mobile nodes with different routing protocols. The graph shows that AODV has a rise in end-to-end delay during the start of simulation because of beacon broadcast, but with the passage of time, it becomes stable and the delay is reduced. Same behavior representation from DSR shows its involvement in cache table addressing. TORA behaved quite stable because of its design to recover a route quickly and that is why it sustains a more stable and an average delay. The end-to end delay varies from 0.020 to 0.025 for AODV after maintaining routing information. DSR started with highest delay of 0.023 , but after 1 minute of simulation, it drops to minimal level of delay. Once AODV and DSR initialized, they easily manage their data traffic and touch the lower limits of End-to-end delay.

But as the number of nodes increase from 10 to 100 , as shown in Figure 3, there are drastic changes. The delay of AODV is almost negligible. With passage of time, the delay of DSR and TORA increases. One interesting observation is that the delay for all protocols increases with larger number of nodes with low mobility, which is due to network congestion. The simulation concludes that the AODV causes lesser delay as compared the other On-Demand protocols.

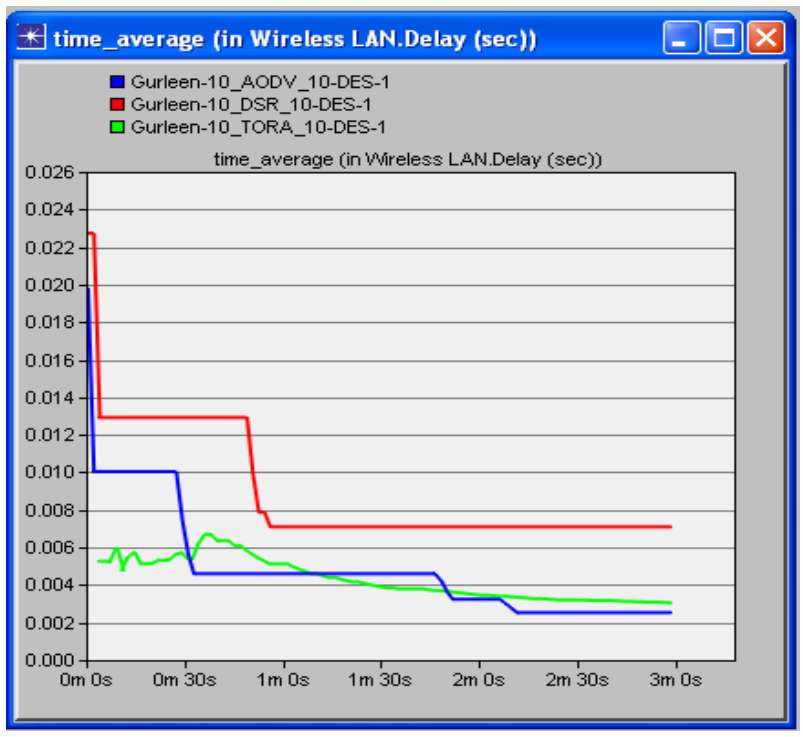

Figure 2. End-to-end Delay for 10 Nodes \& 10m/s Mobility

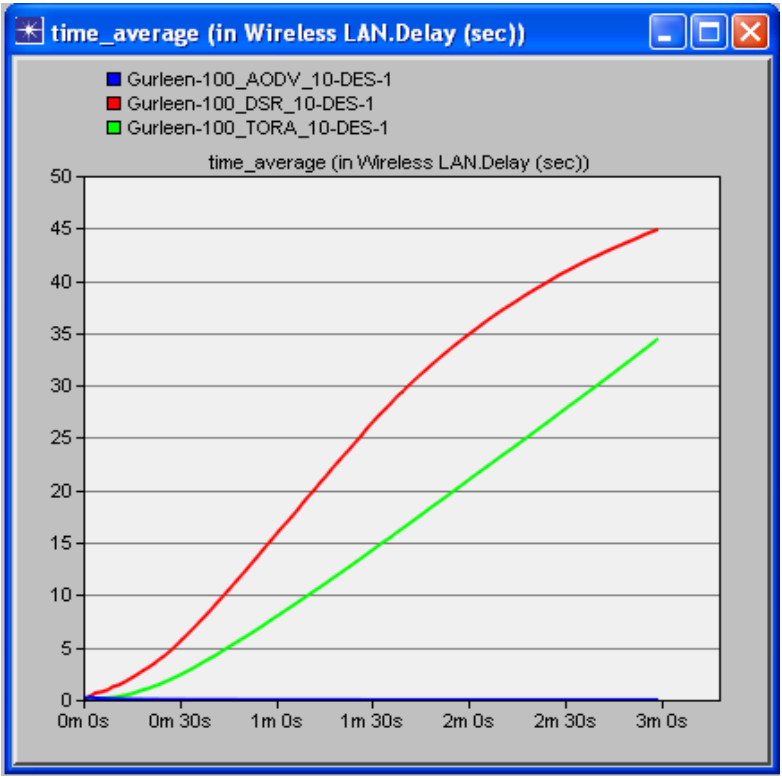

Figure 3. End-to-end Delay for 100 Nodes \& $10 \mathrm{~m} / \mathrm{s}$ Mobility 


\subsubsection{Number of Nodes: 100 and Mobility: 10, 100} $\mathrm{m} / \mathrm{s}$

Figure 4 shows that high mobility has significant effects on delay. The end-to end delay varies from 0.019 to 0.035 for AODV after maintaining routing information. DSR started with highest delay of 0.0215 , but after 1 minute of simulation, it drops to minimal level of delay. TORA behaved quite stable because of its design to recover a route quickly and that is why it sustains a more stable and an average delay.

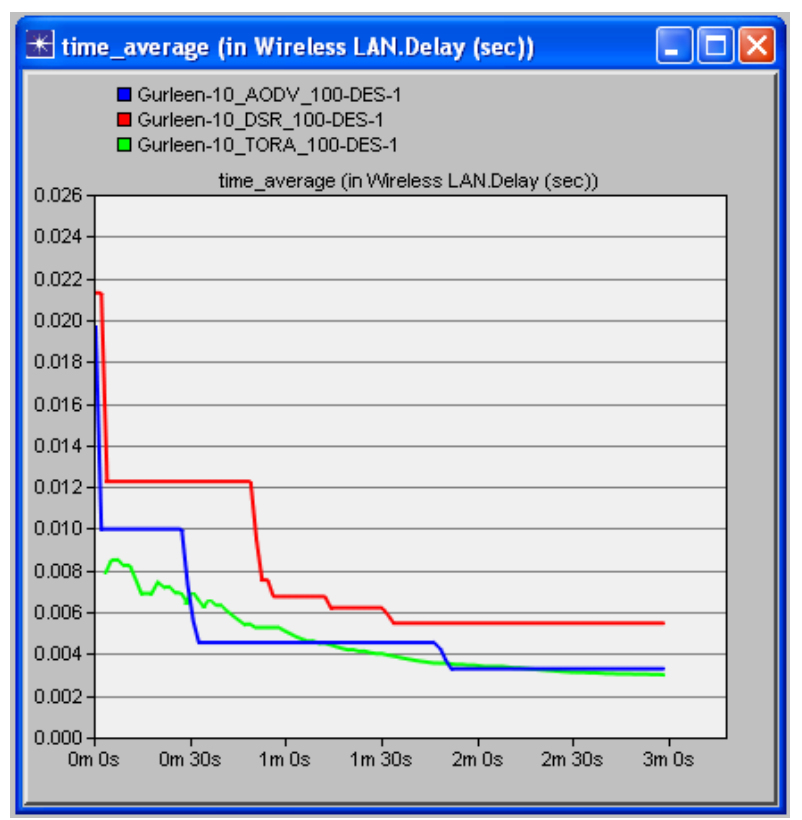

Figure 4. End-to-end Delay for 10 Nodes \& 100m/s Mobility

\subsection{Throughput}

\subsubsection{Number of Nodes: 10, 100 and Mobility: $10 \mathrm{~m} / \mathrm{s}$}

Figure 5 shows the throughput for 10 mobile nodes. The throughput of AODV is maximum but decreases with time. The drop in traffic throughput by DSR and TORA compared to the AODV can be deduced by taking the difference in the traffic dropped by the AODV and the DSR or TORA results in the graph. This dominates the feature of AODV regarding throughput.

But with the increase in number of nodes, as shown in Figure 6 , there are significant changes in the throughput. Throughput of AODV is high in first 30 seconds due to less number of Retransmission Attempts. The throughput for DSR decreases exponentially with time but TORA shows the worst results. But DSR simulation result presents more efficient and better throughput as compared to TORA.

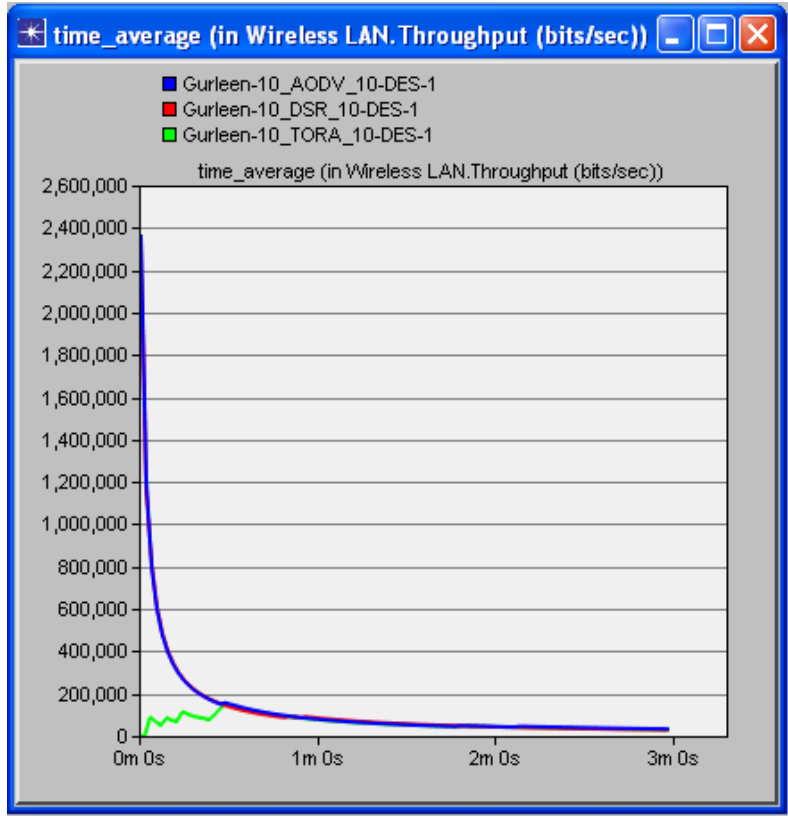

Figure 5. Throughput for 10 Nodes \& 10m/s Mobility

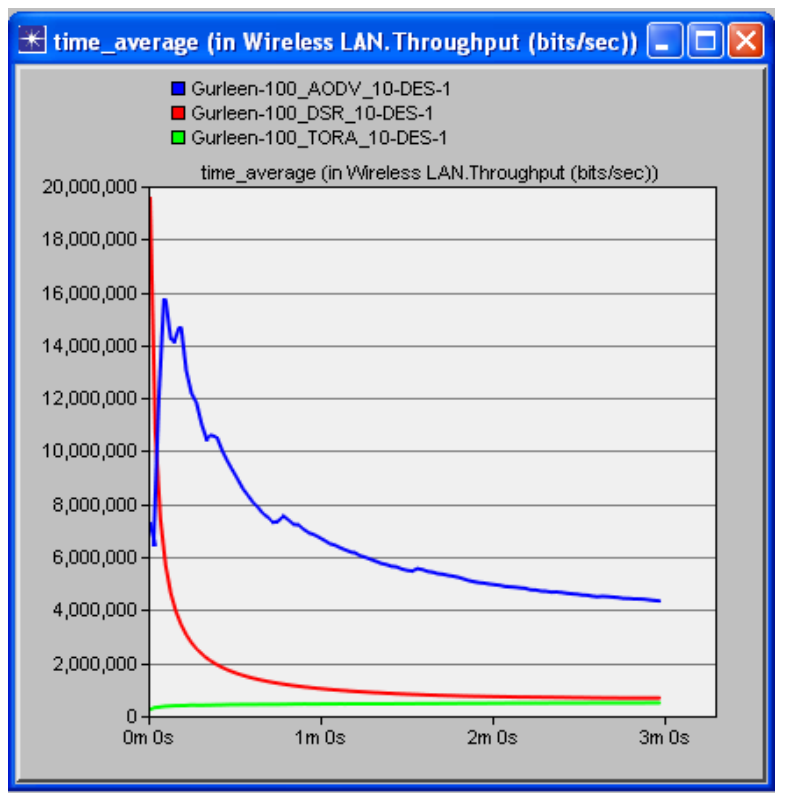

Figure 6. Throughput for 100 Nodes \& $10 \mathrm{~m} / \mathrm{s}$ Mobility

6.2.2 Number of Nodes: 100 and Mobility: 10, 100 $\mathrm{m} / \mathrm{s}$

Figure 7 shows that high mobility has significant effects on throughput. The peak values of throughput of both AODV and DSR decrease with mobility. TORA remains the worst candidate. Hence AODV remains the strong candidate for high throughput. 


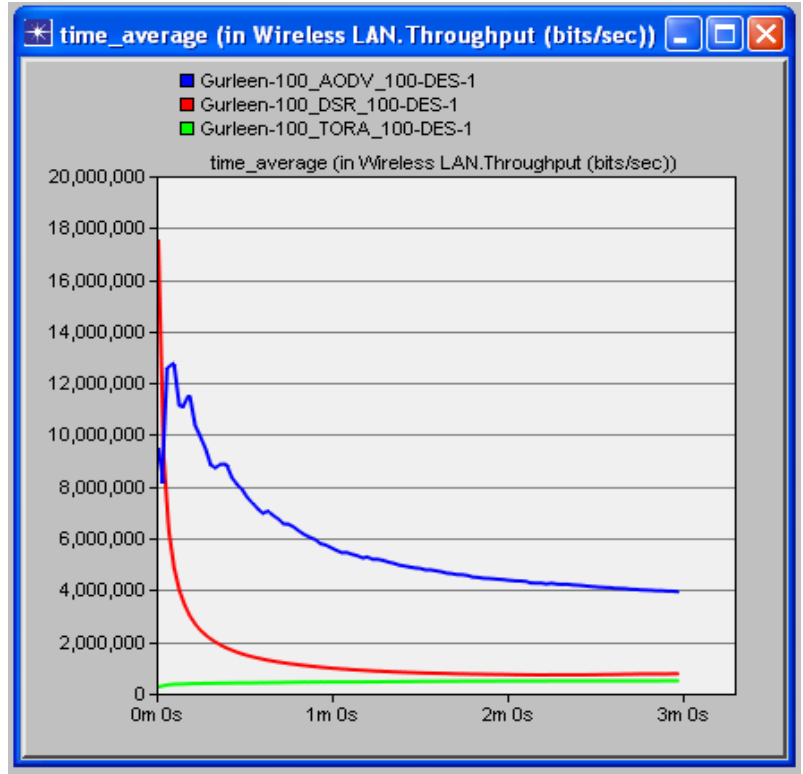

Figure 7. Throughput for 100 Nodes \& 100m/s Mobility

\subsection{Retransmission Attempts}

\subsubsection{Number of Nodes: 10, 100 and Mobility: $10 \mathrm{~m} / \mathrm{s}$}

Figure 8 shows the results of retransmission attempts for 10 nodes. DSR has the lowest value varying from 0.07 to 0.03 and AODV varies from 0.11 to 0.03 . But values for TORA vary from 0.20 to 0.26 . Thus TORA shows the poor results as compared to the other ones.

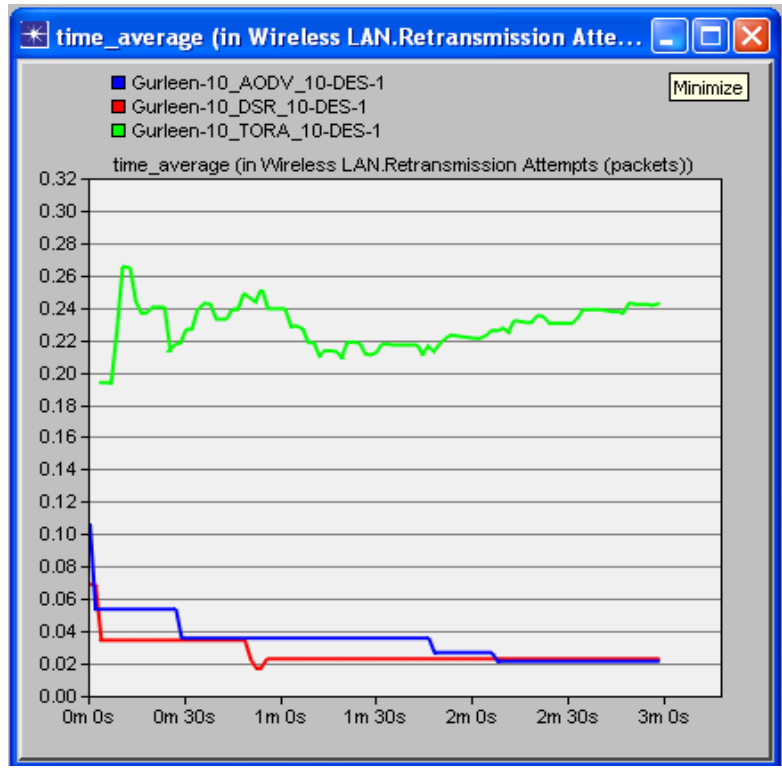

Figure 8. Retransmission Attempts for 10 Nodes \& 10m/s Mobility

With the increase in number of nodes, there are significant changes as shown in Figure 9. There is comparable increase in the values of all the 3 protocols. The value of AODV varies from 0.9 to 0.3 and becomes almost stable. But DSR graph shows an exponential increase with values varying from 0.9 to
1.45. TORA again has the worst results with values varying from 1.4 and 1.6

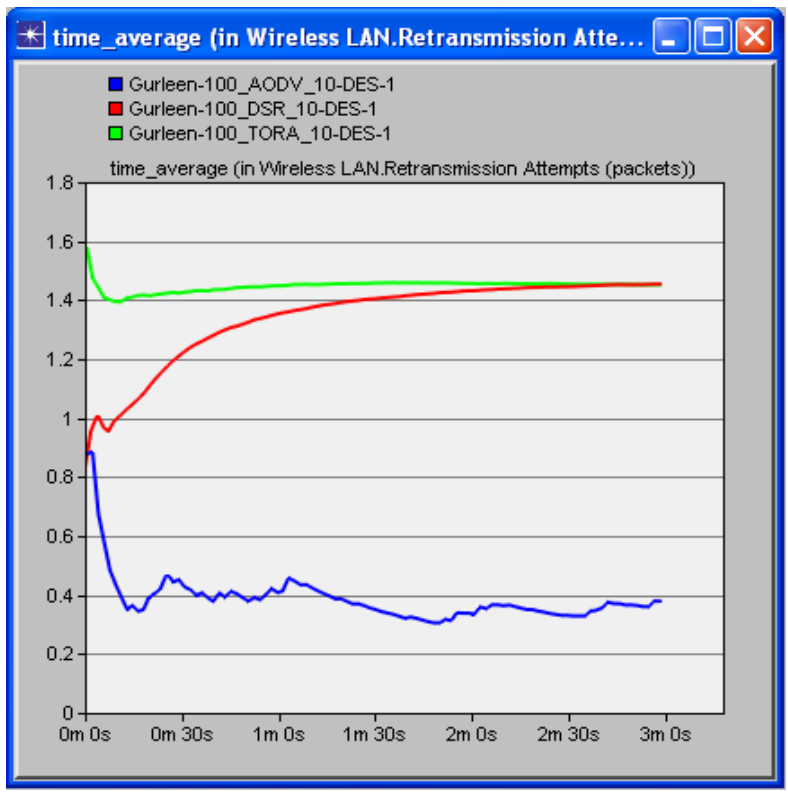

Figure 9: Retransmission Attempts for 100 Nodes \& 10m/s Mobility

6.3.2 Number of Nodes: 10 and Mobility: 10, 100 $\mathrm{m} / \mathrm{s}$

Figure 10 shows the effect on high mobility on retransmission attempts. After 3 minutes of simulation, the graph of AODV and DSR does not merge; rather DSR has more value as opposed to the case with low mobility. Value of TORA varies from 0.335 to 0.245 which is greater than the value with low mobility and shows worst results.

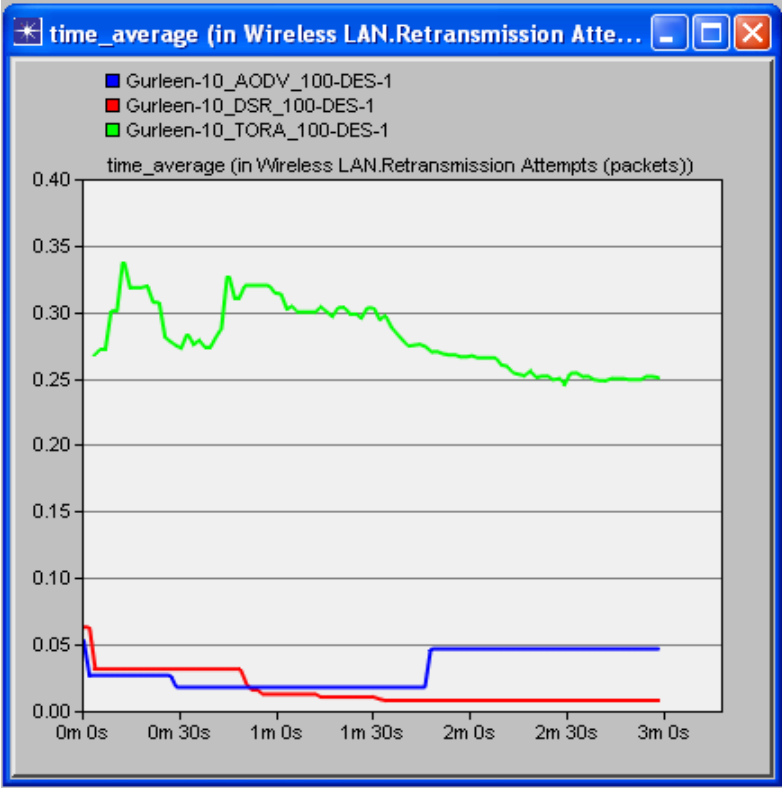

Figure 10. Retransmission Attempts for 10 Nodes \& $100 \mathrm{~m} / \mathrm{s}$ Mobility 


\subsection{Data Dropped}

\subsubsection{Number of Nodes: 10, 100 and Mobility: $10 \mathrm{~m} / \mathrm{s}$}

Figure 11 shows that the data dropped in case of AODV and TORA is almost negligible whereas for DSR, the value is quite high at the start of the simulation but with passage of time, it decreases and becomes almost negligible after 3 minutes.

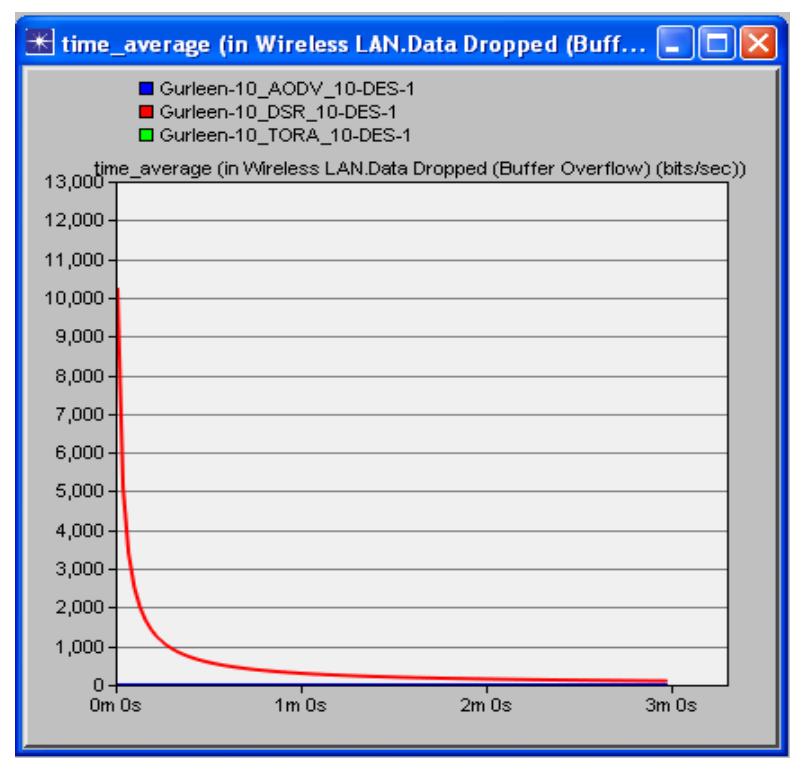

Figure 11. Data Dropped for 10 Nodes \& 10m/s Mobility

With the increase in number of nodes, the results are totally different as shown in Figure 12. Value for TORA is still negligible. But the value for DSR, first of all increases with time and then it starts decreasing gradually after simulation time of 1 minute. AODV has an exponentially decreasing graph. Its value at start of the simulation is very high and becomes almost negligible after 3 minutes.

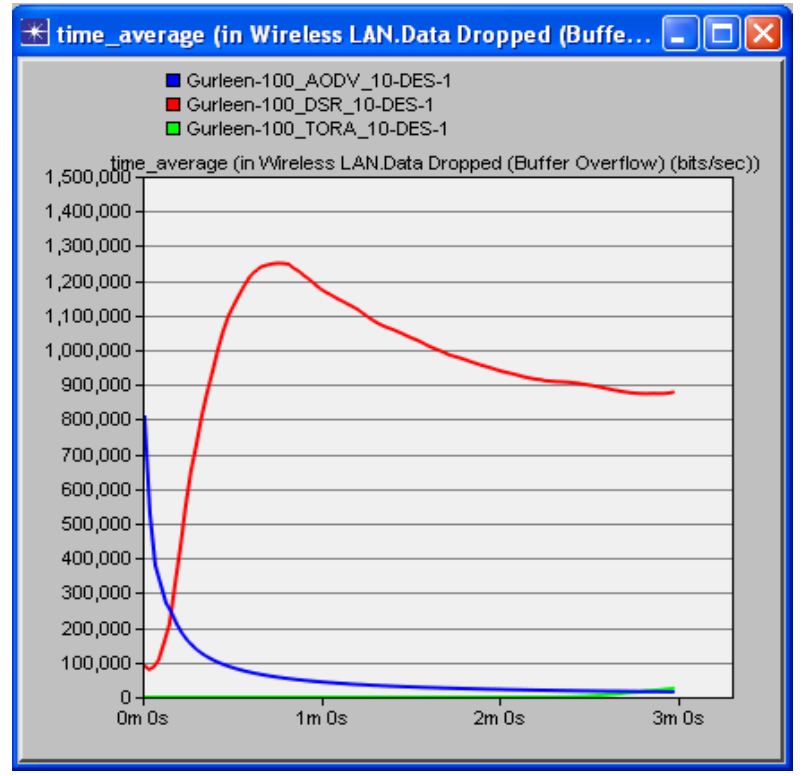

Figure 12. Data Dropped for 100 Nodes \& 10m/s Mobility
6.4.2 Number of Nodes: 100 and Mobility: 10, $100 \mathrm{~m} / \mathrm{s}$

Figure 13 shows the effect of high mobility on the data dropped values. Value for TORA remains negligible till 2 minutes but after that, it starts increasing slowly. Value for AODV is quite less as compared to the value with low mobility. The graph for DSR is also steeper than before.

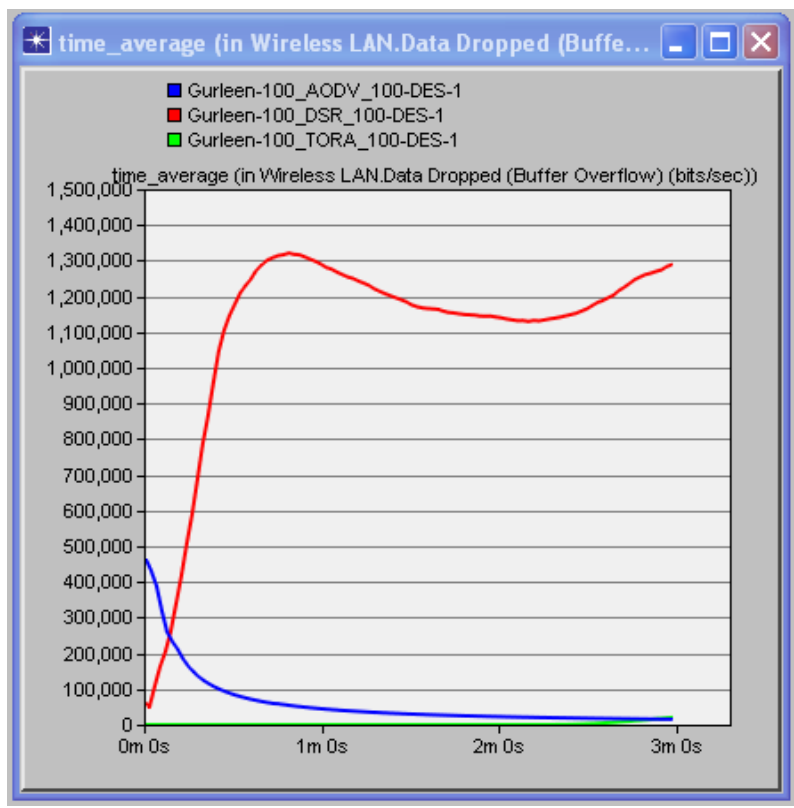

Figure 13. Data Dropped for 100 Nodes \& 100m/s Mobility

\section{ACKNOWLEDGMENTS}

The author would like to thank everyone, just everyone!

\section{CONCLUSIONS}

In this paper, the simulated model scenario compared the performance of AODV, DSR and TORA, the three prominent on-demand routing protocols for ad-hoc networks. A comparison of these three routing protocols is provided, highlighting their features, differences, and characteristics. While no single protocol or class of protocol is the best for all scenarios, each protocol has definite advantages and disadvantages and is well suited for certain situations. Even though AODV, DSR and TORA share on-demand behavior, much of the routing mechanisms are different. In particular, AODV uses routing tables, one route per destination, and destination sequence numbers, a mechanism to prevent loops and to determine freshness of root. On the other hand, DSR uses source routing and route caches and does not depend on any periodic or timer based activity. DSR exploits caching aggressively and maintains multiple routes per destination. TORA provides multiple routes to a destination, establishing a route quickly and minimizing a communication overhead by localizing algorithmic reaction to topological changes. The field of ad-hoc mobile networks is rapidly growing. There are still many challenges that need more attention of researchers. It is likely that such networks will see widespread use within the next few years. 


\section{FUTURE SCOPE}

MANET technology is new emerging technology which has great applications in the new arena of Telecommunication, Internet Systems, and Internet mobility. Routing protocols has the great impact on wireless mesh networks and mobile adhoc networks. Appropriate choosing of protocols according to network increases the credibility and scalability of network. To overcome congestion, traffic load and other restrictions in the network, synchronization of protocols with the network is still challenge for researchers. It is proposed to overcome the drawbacks of proactive routing protocols as well as reactive routing protocols, such as in proactive routing, broadcast of information utilizes more bandwidth and energy in the form of control packets. On the other hand, in reactive routing latency should be overcome which is increased due to route discovery. There are also some other challenges in the form of increase in mobility rate. These kinds of researches should be performed in future to develop reliable and scalable communication in MANETs. Also further simulations and analytical study of protocols with respect to networks should be proceeding for future work.

\section{REFERENCES}

[1] Murthy, C. Sivaram and Manoj, B.S. 2004 Adhoc wireless networks:Architectures, and protocols. Pearson Education.

[2] Broch, J., Maltz, D.A., Johnson, D.B., Hu, Y.C. and Jetcheva, J., "A Performance Comparison of Multi-Hop Wireless Ad-hoc Network Routing Protocols," Proceedings of the 4th Annual ACM/IEEE International Conference on Mobile Computing and Networking (MOBICOM'98), October 1998, pp. 85-97.

[3] Information Sciences Institute, "ns-2 network simulator," Software Package, 2003. [Online]. Available: http://www.isi.edu/nsnam/ns/.

[4] "The VINT Project," USC/ISI, Xerox PARC, LBNL and UC Berkeley, 1997. [Online]. Available: http://www.isi.edu/nsnam/vint/

[5] Cano, J.S. and Manzoni, P., "A Performance Comparison of Energy Consumption for Mobile Ad-hoc Network Routing Protocols", IEEE Transactions, 2000.
[6] Ehsan, H. and Uzmi, Z.A., "Performance Comparison of Ad-hoc Wireless Network Routing Protocols", IEEE Transactions, 2004.

[7] Karthikeyan, N., Palanisamy, V. And Duraiswamy, K., "A Performance Evaluation Of Proactive And Reactive Protocols Using ns-2 Simulation", International J. of Engg. Research \& Indu. Appls. (IJERIA).ISSN 09741518, Vol.2, No.II (2009), pp 309-326

[8] Mobile Ad-hoc Networks URL:http://www.ietf.org/html.charters/manetcharter.html

[9] Johnson, D., Maltz, D. and Hu, Y.C., "The Dynamic Source Routing Protocol for Mobile Ad-hoc Networks," Internet Draft, draft-ietf-manet-dsr- 10.txt, work in progress, July 2004.

[10] Murthy, S. and Garcia-Luna-Aceves, J.J., "An Efficient Routing Protocol for Wireless Networks", ACM Mobile Networks and App. J., Special Issue on Routing in Mobile Communication Networks, Oct. 1996, pp. 18397.

[11] Nesargi, S. and Prakash, R., "A Tunneling Approach to Routing with Unidirectional Links in Mobile Ad-hoc Networks," Proceedings of the IEEE International Conference on Computer Communications and Networks (ICCCN), 2000, pp. 522-527.

[12] Park, V.D. and Corson, M.S., "Temporally-Ordered Routing Algorithm (TORA) Version 1: Functional Specification", Internet draft, draft-ietf-manet-tora- spec04.txt, July 2001.

[13] Temporally Ordered Routing Algorithm URL: http://en.wikipedia.org/wiki/Temporallyordered_routing_algorithm

[14] Jorg, D.O., "Performance Comparison of MANET Routing Protocols in Different Network Sizes", Computer Science Project, University of Berne, Switzerland, 2003

[15] Royer, E.M. and Toh, C.K., "A Review of Current Routing Protocols for Ad-hoc Mobile Wireless Networks", IEEE Personal Communications, Vol. 6, No. 2, pp. 46-55,April1999

[16] Barakovi, S. and Barakovi, J., " Comparative Performance Evaluation of Mobile Ad-hoc Routing Protocols", MIPRO 2010, May 24-28, 2010, Opatija. 